\title{
Wave energy patterns of counterpulsation: A novel approach with wave intensity analysis
}

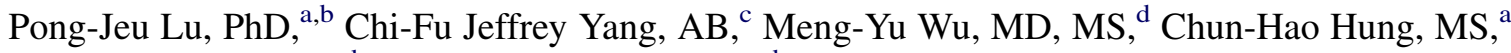 \\ Ming-Yao Chan, $\mathrm{PhD},{ }^{\mathrm{b}}$ and Tzu-Cheng $\mathrm{Hsu}, \mathrm{PhD}^{\mathrm{b}}$
}

\begin{abstract}
Background: In counterpulsation, diastolic augmentation increases coronary blood flow and systolic unloading reduces left ventricular afterload. We present a new approach with wave intensity analysis to revisit and explain counterpulsation principles.
\end{abstract}

Methods: In an acute porcine model, a standard intra-aortic balloon pump was placed in descending aorta in 4 pigs. We measured pressure and velocity with probes in left anterior descending artery and aorta during and without intra-aortic balloon pump assistance. Wave intensities of aortic and left coronary waves were derived from pressure and flow measurements with synchronization correction.

\begin{abstract}
Results: We identified predominating waves in counterpulsation. In the aorta, during diastolic augmentation, intra-aortic balloon inflation generated a backward compression wave, with a "pushing" effect toward the aortic root that translated to a forward compression wave into coronary circulation. During systolic unloading, intraaortic balloon pump deflation generated a backward expansion wave that "sucked" blood from left coronary bed into the aorta. While this backward expansion wave translated to reduced left ventricular afterload, the "sucking" effect resulted in left coronary blood steal, as demonstrated by a forward expansion wave in left anterior descending coronary flow. The waves were sensitive to inflation and deflation timing, with just $25 \mathrm{~ms}$ delay from standard deflation timing leading to weaker forward expansion wave and less coronary regurgitation.
\end{abstract}

Conclusions: Intra-aortic balloon pumps generate backward-traveling waves that predominantly drive aortic and coronary blood flow during counterpulsation. Wave intensity analysis of arterial circulations may provide a mechanism to explain diastolic augmentation and systolic unloading of intra-aortic balloon pump counterpulsation. (J Thorac Cardiovasc Surg 2011;142:1205-13)

The intra-aortic balloon pump (IABP) is the most widely used mechanical circulatory assist device to date, with common clinical indications ranging from cardiogenic shock to postcardiotomy to myocarditis to bridge to transplant. ${ }^{1}$ The IABP is based on the principle of counterpulsation, which has 2 key effects: (1) augmentation of pressure and flow to the coronary circulation during diastole (diastolic augmentation) and (2) reduction of aortic pressure during systole to decrease afterload for ventricular ejection (systolic unloading). ${ }^{1,2}$

\footnotetext{
From the Department of Aeronautics and Astronautics ${ }^{\mathrm{a}}$ and the Heart Science and Medical Devices Research Center, ${ }^{\mathrm{b}}$ National Cheng Kung University, Tainan, Taiwan; Harvard Medical School, ${ }^{\mathrm{c}}$ Harvard University, Boston, Mass; and the Department of Cardiac Surgery, ${ }^{\mathrm{d}}$ Chang Gung Memorial Hospital, Taoyuan, Taiwan.

Supported in part by National Science Council (grant NSC 98-2221-E-006-030) of Taiwan and the University Excellence Fund of National Cheng Kung University. C.-F.Y. was supported by the Howard Hughes Medical Institute Medical Student Fellowship.

Disclosures: Authors have nothing to disclose with regard to commercial support. P-J.L. and C-F.J.Y. contributed equally to this work.

Received for publication Aug 14, 2010; revisions received Jan 29, 2011; accepted for publication Feb 11, 2011; available ahead of print April 8, 2011.

Address for reprints: Pong-Jeu Lu, PhD, Professor of Aeronautics and Astronautics and, Director of Heart Science and Medical Devices Research Center, National Cheng Kung University, 1 University Rd, Tainan, 701, Taiwan (E-mail: pjlu888@yahoo.com).

$0022-5223 / \$ 36.00$

Copyright (C) 2011 by The American Association for Thoracic Surgery

doi:10.1016/j.jtcvs.2011.02.018
}

Studies have shown the IABP to increase peak diastolic and mean coronary flow velocity and to increase blood volume displaced toward the coronary circulation. ${ }^{1-3}$ The physical forces responsible for the coronary flow profile that results from counterpulsation, however, remain poorly understood. Traditional hemodynamic indices of counterpulsation performance-such as vascular pressure changes, the tension time index ${ }^{4}$ (an approximation of myocardial oxygen demand), and the endocardial viability ratio $^{5,6}$ (a measure of myocardial oxygen supply to myocardial oxygen consumption) — cannot elucidate detail in blood flow pulsatility and are unable to describe the dynamic interactions of the heart, the vasculature, and the counterpulsation device.

Wave intensity analysis (WIA) is a time domain analysis introduced by Parker and Jones ${ }^{7}$ that has arisen in recent years as a powerful tool to analyze pulsatile flow circulating in the vascular bed. WIA explains pulsatile flow in terms of a series of infinitesimal, incremental traveling wave fronts that underlie the changes in pressure and flow in arteries. ${ }^{8,9}$ By splitting these wave fronts into "forward" and "backward" components, one can determine the relative contributions of upstream and downstream events. Because it is carried out in the time domain instead of the frequency domain, temporal events in the cardiac cycle can be interpreted directly in terms of energy carried by 


\section{Abbreviation and Acronym \\ $\mathrm{IABP}=$ intra-aortic balloon pump}

wave fronts, or wave intensity, arriving at specific times across a certain fixed cross-section of a vessel, and can be related temporally to hemodynamic parameters on beat-to-beat analyses. ${ }^{9}$ WIA has been used to understand the patterns of flow, wave transmission, and reflection and to quantify the amplitude and direction of energy transport in the systemic, pulmonary, and coronary arterial systems. ${ }^{8,9}$ Recently, it has also been used to examine the aortic wave characteristics and their relationships with counterpulsation therapeutics associated with the IABP. ${ }^{3}$

The purpose of this study was to provide a descriptive view of how waves arise, transmit, and reflect in the ascending aorta and the left coronary vascular bed, particularly as a heart is assisted by IABP counterpulsation. By applying WIA to identify the nature and to quantify the strengths of the waves in the porcine aorta and coronary arteries, we aimed to understand better the device-intervened cardiovascular interaction in the aorta and the complex interaction of proximal and distal influences on left coronary flow during the counterpulsatile support of the IABP.

\section{MATERIALS AND METHODS \\ Animal Model and Instrumentation}

The acute studies were performed on 4 domestic Lanyu minipigs, weighing from 60 to $80 \mathrm{~kg}$. This study was approved by the experimental animal committee of National Cheng Kung University Medical Center, Tainan, Taiwan. The animals received humane care in compliance with the Guide for the Care and Use of Laboratory Animals (NIH publication No. 86-23, revised 1996; www.nap.edu/catalog/5140.html). The animal model, instrumentation, and anesthesia have been previously described. ${ }^{10}$ Briefly, animals were medicated in advance by subcutaneous injection of 6-mg/kg zolazepam hydrochloride-tiletamine hydrochloride (Zoletil) plus $1.5-\mathrm{mg} / \mathrm{kg}$ xylazine (Rompun) and $0.02-\mathrm{mg} / \mathrm{kg}$ atropine. Anesthesia was maintained with isoflurane in oxygen after endotracheal intubation.

Left thoracotomy was performed at the 5 th intercostal space, and the 5 th and 6th ribs were removed to obtain access for the installation of measurement probes. Cannulations were made in the left carotid artery and jugular vein to measure ascending aortic pressure and central venous pressure, respectively, in the right atrial apex to measure the right ventricular pressure, and in the left atrial apex to measure the left ventricular pressure. The left anterior descending coronary artery was dissected free for coronary flow measurement with a Transonic perivascular probe MA 2.5PSS (Transonic Systems Inc, Ithaca, NY). This coronary flow rate was measured approximately $2 \mathrm{~cm}$ downstream of the left anterior descending and circumflex branch juncture. Both aortic and pulmonary flow rates were measured with Transonic probes (MA20PAX).

Datascope Fidelity 8F. 40-cc IAB catheters (Maquet Cardiovascular LLC, Wayne, NJ) were inserted through the left femoral artery according to the standard deployment protocol. The IABP used (Maquet Cardiovascular) had a 40-mL balloon volume and was driven by the Datascope 98 $\mathrm{XT}$ console guided by the skin-measured electrocardiogram. IABPs were intravascularly placed in the descending aortic area. Pumping action was initiated with the Datascope System 98XT console after the IABP insertion had been completed. Other than the monitoring line flushing, no anticoagulant was administered throughout the acute animal experiments.

\section{Data Reduction and Statistical Analysis}

All blood pressure measurements were obtained with a saline solutionfilled pressure transducer system DPT-248 (Utah Medical Products Inc, West Midvale, Utah). We transmitted all acquired pressure and flow rate data into a data acquisition card PCI-6229 (National Instrument, Austin, Texas) driven by the Labview (National Instrument) software system in a personal computer. Postprocessing filter software available in MATLAB (The MathWorks, Inc, Natick, Mass) was adopted to precondition the data. The time lag incurred as a result of the pressure and flow rate measurement site separation and the different data acquisition and filtering systems used was lumped into a time bias constant that was identified and corrected with a special parameter identification method that we developed. ${ }^{11}$

Counterpulsatile support enforced by IABP was carried out in a 1:3 ( 1 pumping assistance per 3 heart beats) fashion. For every 3 heart beats, diastolic augmentation and systolic unloading occurred through the diastolic and systolic phases pertaining to the first and the second beats, respectively; the third beat was taken as the unassisted control beat. Counterpulsatile support effectiveness was evaluated with data taken from the first 2 assisted beats and then compared against those obtained from the third unassisted control beat. Data were phase averaged across 60 beats ( 20 periods) after hemodynamic status stabilized with periodicity established. All time scales of the figures were normalized into $1 \mathrm{~s} / \mathrm{beat}$ to ease the presentation of comparison.

Statistical analysis was performed with SPSS for Windows (version 11.0; SPSS, Inc, An IBM Company, Chicago, Ill). The Student $t$ test was used for the paired data obtained under assisted and control conditions within the same subject group.

\section{Calculation of Wave Intensity}

For an observer located at a certain point of interest in the vascular flow, the pulse observed can be considered a train of wave fronts continuously passing this observer. The net wave intensity, or the energy of a wave front sampled over an infinitesimal time period, is as follows ${ }^{7,8}$ :

$$
d I=d P d U
$$

in which $d P$ and $d U$ are the pressure and velocity differences, respectively, measured across a small time interval defined currently to be $d t=0.004$ seconds. Both $d P$ and $d U$, assumed as $d P=d P_{+}+d P_{-}$and $d U=d U_{+}+$ $d U_{\rightarrow}$ consist of contributions associated with the forward $(+)$ and backward $(-)$ wave modes. Waves can be forward in direction (defined as in the direction of main blood flow) or backward. Further, this wave mode splitting can be determined from the measured pressure and velocity differences. ${ }^{7,8}$

$$
\begin{aligned}
& d P_{ \pm}=\frac{1}{2}(d P \pm \rho c d U) \\
& d U_{ \pm}=\frac{1}{2}\left(d U \pm \frac{d P}{\rho c}\right)
\end{aligned}
$$

in which, $\rho$ is the fluid density and $c$ is the wave speed. The intensities of the forward and backward waves can readily be deduced and expressed by the following equation ${ }^{7,8}$ :

$$
d I_{ \pm}= \pm \frac{1}{4 \rho c}(d P \pm \rho c d U)^{2}
$$

The wave intensity is always positive for the forward-traveling wave and always negative for a backward-traveling wave.

The current aortic or coronary flow velocity was obtained by dividing the measured aortic or left anterior descending coronary flow rate by the 
cross-sectional area of the perivascular probe. The coronary pressure was taken as the nearby ascending aortic pressure corrected with a time delay. Moreover, equations 1 through 4 require the knowledge of the wave speed $c$ to decompose the wave into its associated constituent modes.

\section{Calculation of Wave Speed}

To date, the problem of determining coronary wave speed is unresolved. ${ }^{12-16}$ In this investigation, the wave speed was identified with 2 methods. The first method is the single-point technique that has been previously used in the WIA of coronary circulation in humans subjects. ${ }^{12,15}$ Our second method is a model-based parameter identification method that was recently developed. ${ }^{11}$ When comparing these 2 methods, we found that the calculated average wave speeds in the aorta differed from one another by only $3 \%$. Likewise, the calculated difference in coronary wave speeds was small at 7\%. Thus both methods give the same separation of waves and similar magnitude of peaks when applied to nondiseased arteries (as in the case of this study).

\section{Description of Forward Waves in the Aorta and Coronary Circulation}

In the aorta, forward waves originate from the heart and flow in the direction of left ventricular ejection. ${ }^{9}$ For the coronary arteries, forward wave fronts originate in the left ventricular cavity or the proximal end of the coronary artery, and forward flow is defined as the direction of antegrade perfusion. ${ }^{3}$ Forward waves can be further defined as forward compression waves or forward expansion waves.

Forward compression waves. A forward compression wave is of increasing pressure and velocity with a "pushing" effect. For example, in the ascending aorta, a forward compression wave is generated by left ventricular rapid ejection at early systole. ${ }^{3}$

Forward expansion waves. Forward expansion waves of decreasing pressure and velocity have a "pulling" or "sucking" effect. Therefore, although the direction of the wave is forward, the blood flow velocity is either decreased or in the opposite direction of that defined as forward. For example, during ventricular relaxation, during late systole, the rate of contraction of the ventricle is decreasing, and a forward expansion wave is sent from the ventricular lumen and transmitted through the aorta and along the coronary artery. Although this wave is in the forward direction, sent from the proximal end of the coronary artery to the distal coronary microcirculation, it has a "suction" action-that is, it is a forward suction wave that decreases forward coronary artery blood flow velocity.

\section{Description of Backward Waves in the Aorta and Coronary Circulation}

In the aorta, backward wave fronts arise from reflection in the systemic periphery, whereas in the coronary artery, backward wave fronts originate in the distal coronary microcirculation (the small vessels within the relaxing or contracting myocardium). ${ }^{9}$

Backward wave fronts, like forward wave fronts, can be further categorized as backward compression or backward expansion waves.

Backward compression waves. Backward compression waves, like forward compression waves, increase pressure but cause flow deceleration (flow in the opposite direction of what is defined as forward). ${ }^{9}$ For example, in the aorta, during midsystole backward compression waves arise from reflections of the forward compression waves in the periphery that previously occurred in early systole during left ventricular ejection. ${ }^{3}$ Another example of a backward compression wave occurs during IABP counterpulsation. IABP inflation generates a backward compression wave that increases aortic root pressure and generates backward flow, leading to diastolic aortic pressure augmentation. ${ }^{3}$

Backward compression waves exists in the coronary circulation as well. During early systole before the opening of the aortic valve, ventricular con-
TABLE 1. Hemodynamic performance characteristics of the intraaortic balloon pump group $(n=4)$

\begin{tabular}{|c|c|c|c|}
\hline & Control & IABP & $\begin{array}{c}P \\
\text { value* }\end{array}$ \\
\hline Heart rate (beats/min) & $62 \pm 6$ & $62 \pm 6$ & .965 \\
\hline Mean arterial pressure $(\mathrm{mm} \mathrm{Hg})$ & $57 \pm 3$ & $61 \pm 2$ & .020 \\
\hline End-diastolic aortic pressure $(\mathrm{mm} \mathrm{Hg})$ & $43 \pm 2$ & $37 \pm 5$ & .070 \\
\hline Peak diastolic aortic pressure $(\mathrm{mm} \mathrm{Hg})$ & $56 \pm 2$ & $71 \pm 4$ & .015 \\
\hline $\begin{array}{l}\text { Peak systolic left ventricular } \\
\text { pressure }(\mathrm{mm} \mathrm{Hg})\end{array}$ & $76 \pm 10$ & $70 \pm 12$ & .041 \\
\hline $\begin{array}{l}\text { Peak systolic right ventricular } \\
\text { pressure }(\mathrm{mm} \mathrm{Hg})\end{array}$ & $33 \pm 5$ & $33 \pm 4$ & .675 \\
\hline Stroke volume $(\mathrm{mL})$ & $46 \pm 5$ & $47 \pm 6$ & .201 \\
\hline Cardiac output (L/min) & $2.8 \pm 0.3$ & $2.9 \pm 0.3$ & .227 \\
\hline Coronary perfusion (mL/min) & $37 \pm 7$ & $54 \pm 5$ & .004 \\
\hline Tension time index ${ }^{4}$ & $26 \pm 3$ & $24 \pm 3$ & .006 \\
\hline Endocardial viability ratio ${ }^{5,6}$ & $1.05 \pm 0.11$ & $1.47 \pm 0.28$ & .023 \\
\hline
\end{tabular}

Standard intra-aortic balloon pump served as control. Results are shown as mean \pm SD. Coronary perfusion was defined as the perfused blood volume per heart beat. $I A B P$, Intra-aortic balloon pump. *By Student $t$ test.

traction leads to compression of the distal vessels in the coronary microcirculation, as described by the intramyocardial pump model. ${ }^{3}$ This generates a compression wave (a wave that increases pressure and pushes). This wave is a backward compression wave because it originates from the microcirculation, travels backwards along the coronary artery, and pushes towards the coronary ostium. $^{16}$

Backward expansion waves. In contrast, the backward expansion wave decreases pressure but causes flow acceleration (the backward expansion wave sucks in the direction opposite to that which is defined as forward). ${ }^{3}$ Thus the direction of the flow is the same as that of a forward compression wave - that is, both the forward compression wave and the backward expansion wave accelerate flow in the forward direction. For example, during systolic unloading, IABP deflation generates a backward expansion wave that causes a decrease in aortic root pressure, which generates a suction wave (a pulling effect away from the heart) and an increase in forward flow.

\section{RESULTS}

\section{Hemodynamic Parameters}

The classic quantification of systolic unloading by the decrease of end-diastolic aortic pressure or peak systolic left ventricular pressure as well as the measure of diastolic augmentation by the increase of the peak diastolic aortic pressure were demonstrated in these IABP experiments by comparing the assisted and unassisted beats (Table 1).

\section{Aortic Wave Intensities}

Figure 1 shows the aortic pressure and flow rate measurements and the deduced wave intensities of a representative IABP-assisted subject with a standard inflation-deflation control setting of inflation at the dicrotic notch and a deflation of $25 \mathrm{~ms}$ before the $\mathrm{R}$ wave. Pressure, velocity, and wave intensity recordings obtained in the ascending aorta were decomposed into contributions associated with the positive (heading downstream) and negative (heading 

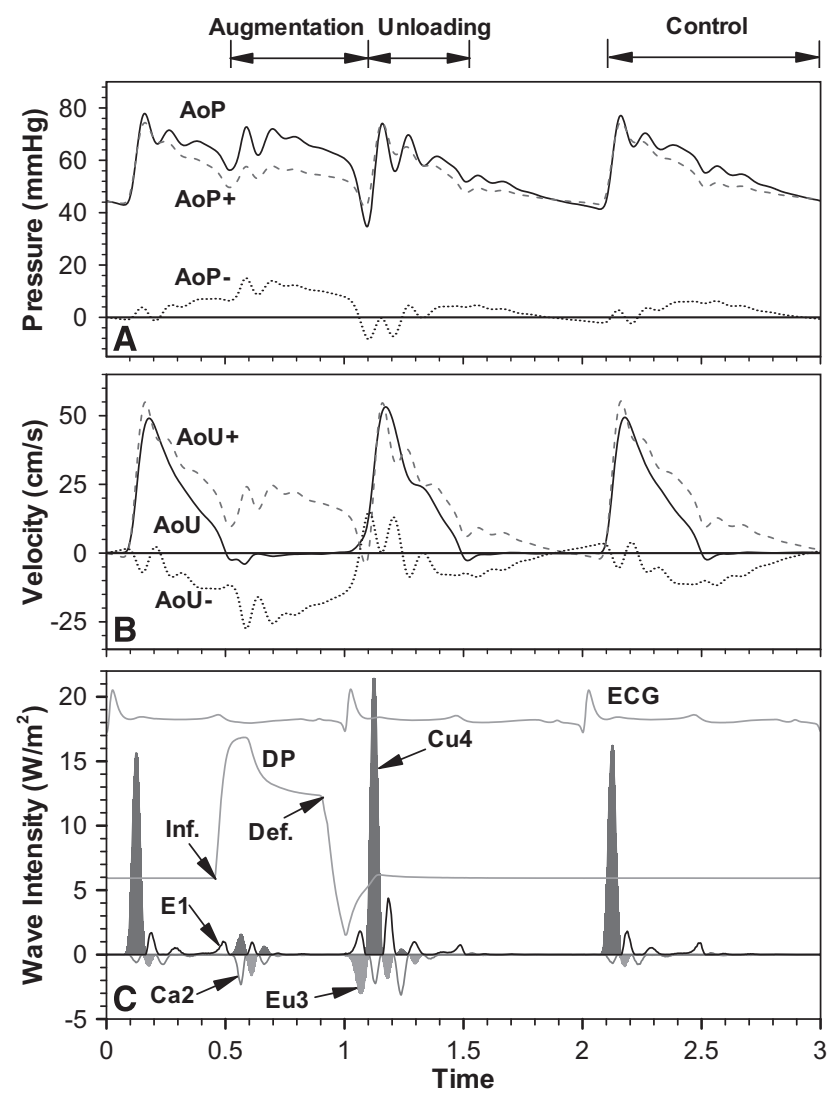

FIGURE 1. Aortic hemodynamic and wave intensity characteristics under intra-aortic balloon pump counterpulsatile support timed at standard inflation-deflation setting. Inflation-deflation $0 /-1$; inflation instant 0 denotes inflation at the dicrotic notch, and deflation instant -1 denotes deflation at 1 count $(25 \mathrm{~ms}$ ) preceding the $\mathrm{R}$ wave. A, Aortic pressure (AoP, solid line) and its decomposed wave components $\mathrm{AoP}_{+}$(short-segment line) and $\mathrm{AoP}_{-}$(dotted line). $\mathrm{B}$, Aortic flow velocity (AoU, solid line) and its decomposed wave modes $\mathrm{AoU}_{+}$(short-segment line) and $\mathrm{AoU}_{-}$(dotted line). $\mathrm{C}$, Wave intensities $\left(\mathrm{dI}_{ \pm}, \mathrm{dL}_{+}>0\right.$ and $\mathrm{dL}<0$; shaded areas indicate acceleration waves with $\mathrm{dU}_{ \pm}>0$, whereas waves and hollow areas indicate deceleration waves with $\mathrm{dU}_{ \pm}<0$ ). Symbols appearing in the wave intensity panel are prominent waves associated with counterpulsation: capital letters $C$ and $E$ represent compression or expansion wave, with the numeric value indicating the occurrence sequence in time history; subscripts $a$ and $u$ under $\mathrm{C}$ and $\mathrm{E}$ signify intra-aortic balloon pump-generated or -influenced waves as a result of diastolic augmentation (denoted with subscript $a$ ) or systolic unloading (denoted with subscript $u$ ). $E C G$, Electrocardiogram; $D P$, drive line pressure; Inf., inflation timing denoted on the DP waveform; Def., deflation timing denoted on the DP waveform.

upstream) wave components. In Figure $1, C$, there are 4 intensity peaks (marked with $E 1, C_{a} 2, E_{u} 3$, and $C_{u} 4$ ) that are essential to the counterpulsation performance. Upper case letters $C$ and $E$ denote the compression (pushing) or expansion (pulling or sucking) nature of the wave, respectively, and the numeric value represents the sequence in which the waves arise. The subscripts $a$ and $u$ denote IABP-generated and IABP-influenced waves associated with diastolic augmentation (denoted with subscript $a$ ) or systolic unloading (denoted with subscript $u$ ). It is seen that backward $\mathrm{C}_{\mathrm{a}} 2$ and $\mathrm{E}_{\mathrm{u}} 3$ waves are IABP generated as a result of balloon inflation and deflation, respectively, whereas $\mathrm{C}_{\mathrm{u}} 4$ corresponds to the accelerated systolic ejection caused by the balloon-induced contraction unloading.

\section{Left Coronary Wave Intensities}

The unassisted control beat. We first examined as a benchmark the coronary flow characteristics of the unassisted control beat (the third beat $\mathrm{T}=2.0 \sim 2.6$; Figure 2, $C$ ) associated with the aortic flow and wave intensities shown in Figure 1. For an unassisted beat, major left ventricular contraction-relaxation and aortic pressurization-depressurization activities were characterized by peaks shown on the wave intensity traces, and the prominent events were denoted E1, C2, C3, E4, E5, and C6 ( T = 2.0 2.6) on the figure. In the discussion of coronary flows, antegrade coronary flow perfused into myocardium was defined as forward traveling, and retrograde flow (originating from the distal microcirculation) as backward traveling.

Around left ventricular end diastole, the aortic pressure dropped, while flow decelerated and resulted in a forward expansion E1 wave sent through the aortic ostium into the left coronary vasculature. Similar to the situation found in the human coronary circulation, ${ }^{12}$ a backward $\mathrm{C} 2$ compression wave immediately followed. This $\mathrm{C} 2$ wave was produced by left ventricular isovolumetric contraction that propelled blood retrograde from the myocardial microcirculation toward the epicardial arteries. This isovolumetric contraction quickly increased left ventricular pressure, resulting in aortic valve opening. Arterial pressure soared on aortic valve opening, generating a $\mathrm{C} 3$ forward compression wave to the coronary bed.

In late systole, left ventricular relaxation caused both aortic pressure and intramyocardial compression to decline. The deceleration of aortic flow in late systole generated the E4 forward-traveling expansion wave, which was sent into the left coronary vessels from the aortic ostium. The E5 backward-traveling expansion wave was created in the distal coronary vasculature bed, corresponding to myocardial microcirculatory decompression and ventricular relaxation. The $\mathrm{C} 6$ forward-traveling compression wave, located around the dicrotic notch, arose at the moment that the aortic valve closed, when the aortic pressure was briefly increased.

Except for the initial systolic C3 forward-traveling compression wave that drove blood flow into the left coronary arteries, it was mainly in the diastolic phase that blood was seen to be perfused into the left coronary vasculature. Diastolic augmentation. Diastolic augmentation provided by IABP is illustrated in the first beat of Figure 2 $(\mathrm{T}=0.45 \sim 0.8)$. Forward expansion wave $\mathrm{E} 4$ was first generated in late systole as a result of aortic flow deceleration 


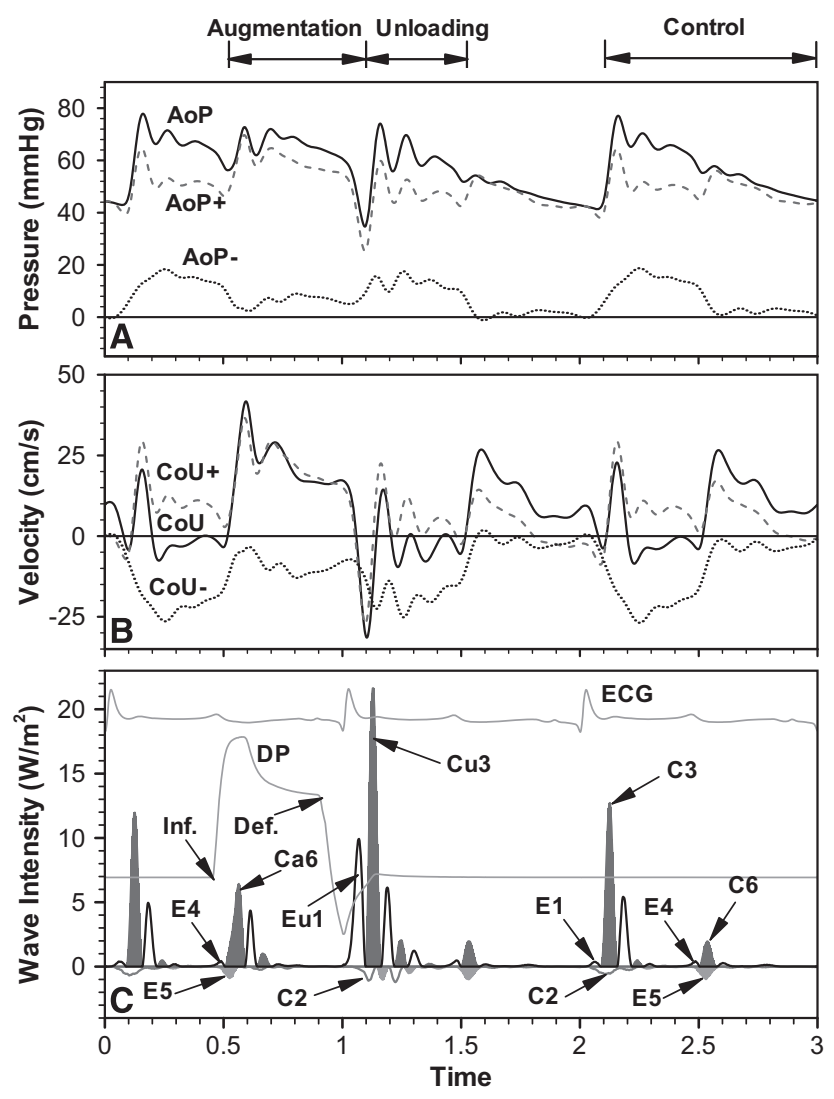

FIGURE 2. Left coronary hemodynamic and wave intensity characteristics under intra-aortic balloon pump counterpulsatile support timed at standard inflation-deflation setting. Inflation-deflation $0 /-1$; inflation instant $O$ denotes inflation at the dicrotic notch, and deflation instant -1 denotes deflation at 1 count $(25 \mathrm{~ms})$ preceding the $\mathrm{R}$ wave. A, Aortic pressure (AoP, solid line) and its decomposed wave components $\mathrm{AoP}_{+}$(short- segment line) and $\mathrm{AoP}_{-}$(dotted line). $\mathrm{B}$, Coronary flow velocity ( $\mathrm{CoU}$, solid line) and its decomposed wave modes $\mathrm{CoU}_{+}$(short-segment line) and $\mathrm{CoU}_{-}$ (dotted line). $\mathrm{C}$, Wave intensities $\left(\mathrm{dI}_{ \pm}, \mathrm{dL}_{+}>0\right.$ and $\mathrm{dL}<0$; shaded areas indicate acceleration waves with $\left(\mathrm{dU}_{ \pm}>0\right)$ and hollow areas indicate deceleration waves with $\mathrm{dU}_{ \pm}<0$ ). Symbols appearing in the wave intensity panel are prominent waves associated with counterpulsation: capital letters $C$ and $E$ represent compression or expansion wave, with the numeric value indicating the occurrence sequence in time history; subscripts $a$ and $u$ under $\mathrm{C}$ and $\mathrm{E}$ signify intra-aortic balloon pump-generated or -influenced waves from diastolic augmentation (denoted with subscript $a$ ) or systolic unloading (denoted with subscript $u$ ). $E C G$, Electrocardiogram; $D P$, drive line pressure; Inf., inflation timing denoted on the DP waveform; Def., deflation timing denoted on the DP waveform.

before valve closure. The E5 suction wave that originated in the distal microcirculation remained unchanged in strength when compared with the E5 wave of the control state because augmentation came from the proximal aortic end. IABP inflation generated the forward compression wave $\mathrm{C}_{\mathrm{a}} 6$. Corresponding with this elevated $\mathrm{C}_{\mathrm{a}} 6$ wave intensity was an increase in pressure and perfusion velocity, as shown in panels $A$ and $B$ of Figure 2.
Systolic unloading. Systolic unloading effect is illustrated in the coronary waveform profiles revealed around the unloading period ( $\mathrm{T}=1.0 \sim 1.3$; Figure 2$)$. Unloading was initiated before aortic valve opening, as indicated by the deflation-induced $\mathrm{E}_{\mathrm{u}} 1$ forward expansion wave sent from the proximal coronary entrance. Compared with the control case, this unloading $\mathrm{E}_{\mathrm{u}} 1$ forward expansion (pulling) wave was much strengthened. The ensuing $\mathrm{C} 2$ backward compression wave, corresponding to the retrograde intramyocardial pumping wave, appeared during the isovolumetric contraction period of the ventricle. Subsequent cardiac systole and aortic valve opening elevated the aortic pressure and generated the $\mathrm{C}_{\mathrm{u}} 3$ forward-traveling compression wave.

\section{Inflation-Deflation Control Effect}

Figures 3 and 4 show the aortic and left coronary wave intensities during IABP counterpulsation with a different deflation timing - this time, instead of deflating 1 count ( $25 \mathrm{~ms}$ ) before the $\mathrm{R}$ wave (Figures 1 and 2), the balloon deflation timing was delayed slightly to the right at the $\mathrm{R}$ wave. In the aorta, the wave intensities $\mathrm{E}_{\mathrm{u}} 3$ and $\mathrm{C}_{\mathrm{u}} 4$, corresponding to systolic unloading, were weaker in this $\mathrm{R}$-wave deflation setting (Figure 3, $C$ ) than in the standard setting (Figure 1,C). Similarly, in the coronary wave intensities (Figure 4,C), it can be seen that all the unloading-related wave intensities $\left(\mathrm{E}_{\mathrm{u}} 1\right.$ and $\mathrm{C}_{\mathrm{u}} 3$, around $\mathrm{T}=1.0 \sim 1.4$ ) were weaker in the R-wave deflation setting than in the standard setting.

In the left coronary circulation, during the R-wave deflation setting (Figure 4), there was a weaker $\mathrm{E}_{\mathrm{u}} 1$ wave than was seen in the standard timing. This weaker $\mathrm{E}_{\mathrm{u}} 1$ wave corresponded to reduced coronary regurgitation at time of systolic unloading, as shown in the coronary flow velocity curves (Figure 4, $B$ ).

\section{DISCUSSION}

In this study, we first evaluated IABP counterpulsation with traditional performance indices. We found the IABP to be successful in providing diastolic augmentation and systolic unloading.

Next, we introduced WIA to assess the counterpulsation performance of the IABP. With WIA, we have not discovered any new biophysical phenomena but rather have introduced a method of analysis that can be used to assess counterpulsation with higher resolution than previous techniques. Unlike traditional methods of examining counterpulsation, such as pressure changes or cardiac output, WIA allows temporal events in the cardiac cycle to be interpreted directly in terms of energy carried by wave fronts or wave intensity.

Kolyva and colleagues ${ }^{3}$ recently demonstrated with WIA that inflation and deflation of the IABP generated in the 

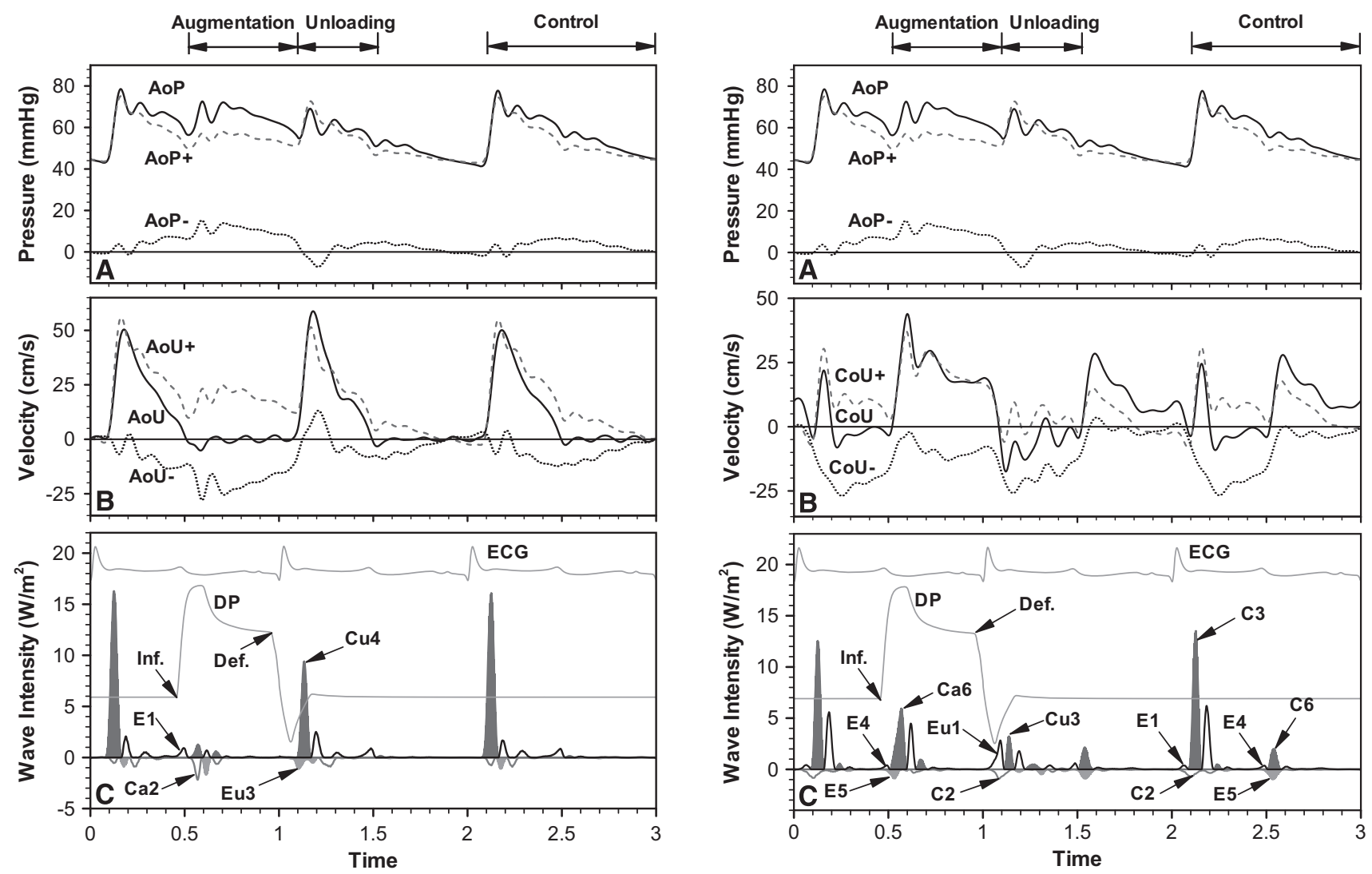

FIGURE 3. Aortic hemodynamic and wave intensity characteristics under intra-aortic balloon pump counterpulsatile support timed at R-wave deflation. Inflation-deflation $0 / 0$; inflation instant $O$ denotes inflation at the dicrotic notch, and deflation instant $O$ denotes deflation at the $\mathrm{R}$ wave. A, Aortic pressure (AoP, solid line) and its decomposed wave components $\mathrm{AoP}_{+}$(short-segment line) and $\mathrm{AoP}_{-}$(dotted line). B, Aortic flow velocity (AoU, solid line) and its decomposed wave modes $\mathrm{AoU}_{+}$(short-segment line) and AoU_ (dotted line). $\mathrm{C}$, Wave intensities $\left(\mathrm{dI}_{ \pm}, \mathrm{dI}_{+}>0\right.$ and $\mathrm{dL}<0$; shaded areas indicate acceleration waves with $\mathrm{dU}_{ \pm}>0$ and hollow areas indicate deceleration waves with $\mathrm{dU}_{ \pm}<0$ ). Symbols appearing in the wave intensity panel are prominent waves associated with counterpulsation: capital letters $C$ and $E$ represent compression or expansion wave, with the numeric value indicating the occurrence sequence in time history; subscripts $a$ and $u$ under $\mathrm{C}$ and $\mathrm{E}$ signify intra-aortic balloon pump-generated or -influenced waves as a result of diastolic augmentation (denoted with subscript $a$ ) or systolic unloading (denoted with subscript $u$ ) effect. $E C G$, Electrocardiogram; $D P$, drive line pressure; Inf. inflation timing denoted on the DP waveform; Def., deflation timing denoted on the DP waveform.

aorta a backward compression wave and a backward expansion wave, respectively, and that the energies of these waves were directly correlated with peak diastolic pressure elevation and end-diastolic pressure reduction. They were unable to obtain coronary flow measurements, however, and establish the WIA for coronary circulation with the IABP assist.

Specifically, we have expanded on the previous research by demonstrating how WIA can be used to identify and quantify the prominent waves in the left coronary artery and aorta

FIGURE 4. Left coronary hemodynamic and wave intensity characteristics under intra-aortic balloon pump counterpulsatile support timed at Rwave deflation. Inflation-deflation $0 / 0$; inflation instant $O$ denotes inflation at the dicrotic notch, and deflation instant $O$ denotes deflation at the $\mathrm{R}$ wave. A, Aortic pressure (AoP, solid line) and its decomposed wave components $\mathrm{AoP}_{+}($short-segment line $)$and $\mathrm{AoP}_{-}($dotted line $) . \mathrm{B}$, Coronary flow velocity ( $\mathrm{CoU}$, solid line) and its decomposed wave modes $\mathrm{CoU}_{+}$(short-segment line) and $\mathrm{CoU}_{-}$(dotted line). $\mathrm{C}$, Wave intensities $\left(\mathrm{dI}_{ \pm}, \mathrm{dI}_{+}>0\right.$ and $\mathrm{dI}<0$; shaded areas indicate acceleration waves with $\mathrm{dU}_{ \pm}>0$ ) and hollow areas indicate deceleration waves with $\mathrm{dU}_{ \pm}<0$ ). Symbols appearing in the wave intensity panel are prominent waves associated with counterpulsation: capital letters $C$ and $E$ represent compression or expansion wave, with the numeric value indicating the occurrence sequence in time history; subscripts $a$ and $u$ under $\mathrm{C}$ and $\mathrm{E}$ signify intra-aortic balloon pump-generated or -influenced waves as a result of diastolic augmentation (denoted with subscript $a$ ) or systolic unloading (denoted with subscript $u$ ) effect. $E C G$, Electrocardiogram; $D P$, drive line pressure; Inf., inflation timing denoted on the DP waveform; Def., deflation timing denoted on the DP waveform.

in an acute porcine model during IABP assistance. Second, we have shown how components associated with forwardand backward-traveling waves are sensitive to even minute changes in inflation-deflation timing adjustment.

\section{Aortic Wave Intensities}

Diastolic augmentation and systolic unloading. Diastolic augmentation can be visualized by the wave intensities situated around the dicrotic notch $(\mathrm{T}=0.5 \sim 0.7$; Figures 1 and 3). Before aortic valve closure, decelerated 
aortic flow sent downstream a forward expansion wave E1; immediately after this time, the IABP was inflated. The main wave created on inflation of the IABP during diastole was the $\mathrm{C}_{\mathrm{a}} 2$ backward compression wave. This wave traveled from the balloon backward toward the aortic root to push blood flow into the coronary bed.

The main wave corresponding to systolic unloading in the aorta was characterized by the $\mathrm{E}_{\mathrm{u}} 3$ backward expansion wave ( $\mathrm{T} \sim 1.07$ in Figure 1, $C$, standard deflation, and $\mathrm{T} \sim 1.1$ in Figure 3, $C$, R-wave deflation) that was generated by pump deflation. $\mathrm{C}_{\mathrm{u}} 4$ was the subsequent forward compression wave generated by left ventricular ejection. These wave patterns are consistent with those of previous human IABP support. ${ }^{3}$

Effect of timing adjustment. The aortic wave intensities were sensitive to the timing of the balloon deflation. When the timing was delayed to deflation right at the $\mathrm{R}$ wave (also known as R-wave deflation) as opposed to 25 $\mathrm{ms}$ before the $\mathrm{R}$ wave (which was the standard timing), there were significant changes in wave intensity strength. In the R-wave deflation setting, the systolic $\mathrm{C}_{\mathrm{u}} 4$ compression wave intensity $\mathrm{dL}_{+}(\mathrm{T}=1.1 \sim 1.2$, Figure $3, C)$ was $40 \%$ smaller than the left ventricular systolic ejection wave intensity of an unassisted beat $(\mathrm{T}=2.1$, Figure 3 , C). The $\mathrm{E}_{\mathrm{u}} 3$ expansion (suction) wave generated by pump deflation was also weaker (dL diminished by $67 \%$ ) than that seen in the standard timing unloading. This is because the balloon deflation timing was such that the onset of the left ventricular ejection $\mathrm{C}_{\mathrm{u}} 4$ compression wave and the $\mathrm{E}_{\mathrm{u}} 3$ unloading expansion wave were so close to each other that they overlapped and thus canceled each other out to a significant extent.

In contrast, for the standard deflation control setting ( 25 $\mathrm{ms}$ before the $\mathrm{R}$ wave), the balloon was fully deflated earlier and occupied less space in the aorta for a longer period during systole. The IABP had longer time to deplete the helium contained in the balloon before aortic valve opening, resulting in more effective afterload reduction to help the subsequent left ventricular systolic ejection. In the ascending aorta (Figure 1,C), an intensified $\mathrm{E}_{\mathrm{u}} 3$ backward expansion wave was observed; this meant that a greater suction wave effect and greater afterload reduction were induced by the IABP. The subsequent systolic $\mathrm{C}_{\mathrm{u}} 4$ forward compression wave was intensified as a result of accelerated forward flow because the left ventricle was pumping blood against a reduced afterload.

Although greater systolic unloading was achieved, there was a trade-off. Earlier IABP deflation often caused retrograde left coronary flow (coronary blood steal), as shown by the coronary WIA in the following discussion.

\section{Left Coronary Wave Intensity}

Unassisted control. The coronary flow profile without mechanical circulatory assistance has been previously elucidated for the canine ${ }^{17}$ and human ${ }^{16}$ left coronary circulation. Interestingly, the wave intensities in our unassisted beat closely resembled those of the human coronary circulation; the major waves that we identified in the unassisted condition are also found in the human coronary circulation.

The main difference between our porcine model and the human circulation lies in the magnitude of the E5 expansion wave. In the human circulation, this wave, commonly referred to as the backward-traveling suction wave, is the dominant perfusion force that sucks coronary blood from the aorta during diastole. ${ }^{16}$

In our porcine model, E5 suction wave strength was greatly suppressed relative to that in the human circulation. $^{16}$ The cause for this inconsistency may have been a difference in study methods. In the study of Davies and coworkers ${ }^{16}$ the chests of the patients were intact, whereas in our experiments a left thoracotomy with 5th and 6th rib removal was performed. In this study the pig's chest was left open to allow incorporation of necessary pressure and flow rate instruments. In this situation, the exposed heart became dilated, with end-systolic and end-diastolic left ventricular volumes increasing $13 \%$ and $16 \%$, respectively. This dilation may have affected the degree of muscular relaxation and resulted in the weakening of the myocardial relaxation or coronary suction force generation in late systole. This finding is consistent with the previous human data. Of note, the weakened E5 suction waves have previously been found in patients with left ventricular hypertrophy and impairment of left ventricular diastolic relaxation. ${ }^{16}$

Diastolic augmentation and systolic unloading. The main diastolic augmentation wave was the $C_{a} 6$ forward compression wave. This $\mathrm{C}_{\mathrm{a}} 6$ wave was generated by the IABP pumping, which compressed (pushed) blood flow to the left coronary circulation. Generally, the $\mathrm{C}_{\mathrm{a}} 6$ wave was trailed by a forward expansion wave. This trailing expansion wave decelerated the antegrade coronary flow, which is a commonly seen residue occurring in all flow compression processes. Note that the coronary $\mathrm{C}_{\mathrm{a}} 6$ and E5 waves overlapped a great portion in time.

During this push-and-pull diastolic period, greater left coronary perfusion was achieved as a result of the larger pressure gradient contributed together by antegrade $C_{a} 6$ augmenting and retrograde E5 suction waves, working on a more relaxed myocardium of reduced resistance, in the left coronary bed.

The main wave generated by systolic unloading was the deflation-induced $\mathrm{E}_{\mathrm{u}} 1$ forward expansion wave sent from the proximal coronary entrance. Relative to the control case (the unassisted beat), this unloading $\mathrm{E}_{\mathrm{u}} 1$ forward expansion wave was greatly strengthened and was accompanied a prominent "dip" in left anterior descending coronary flow velocity profile (Figure $2, B$ ), indicating that the IABP deflation generated a suction wave that sucked blood out of the left coronary circulation. 
Effect of timing adjustment. In the previous discussion of the WIA of the aorta, we noted that standard deflation timing (deflation 1 count, $25 \mathrm{~ms}$, before the $\mathrm{R}$ wave) resulted in a much stronger systolic unloading wave and greater left ventricular afterload reduction. This corresponded with a stronger $E_{\mathrm{u}} 1$ forward expansion wave in the left coronary circulation, indicating that there was greater suction of the coronary blood flow from the aorta in the standard deflation timing than in the R-wave deflation timing.

Systolic unloading design is complex because it entails contradictory influences in the regaining of the supply-demand equilibrium for diseased myocardium. ${ }^{2,5,6}$ Lowering the end-diastolic pressure allows easier ventricular ejection and relieves the pathologically stressed myocardium. This left ventricular decompression contributes to an improved perfusion in the left ventricular endomyocardium and helps the infarcted myocyte to recover. An excessively low enddiastolic aortic pressure, however, may incur severe coronary regurgitation ${ }^{18}$ before left ventricular systole, which may jeopardize the desired overall coronary perfusion augmentation. We demonstrated here that the left coronary regurgitation from IABP deflation is generated by a strong pump-induced forward expansion suction wave.

\section{Potential WIA Applications}

In the future, wave intensities could be used as a monitoring signal to fine-tune counterpulsation performance. Practicing clinicians will likely prefer automatic implementation of WIA as part of the control system of the IABP. To this end, there will need to be easily obtainable and reliable noninvasive, real-time pressure and velocity measurements. Recent progress has been made with noninvasive acquisition of pressure and flow velocity data and the associated real-time forward-backward wave intensity presentations. $^{21,22}$

In addition to signal gathering and processing, future research for WIA should involve understanding the therapeutic efficacy of counterpulsation-generated compression and expansion waves. WIA, wave transmission-reflection, and wave speed determination also could be used to assess how counterpulsation is affected by such pathologic entities as coronary stenosis, atherosclerosis, microcirculatory disease, and abnormalities of ventricular function.

\section{Study Limitations}

This study of coronary perfusion augmentation by IABP counterpulsation was focused on the left ventricle. The right ventricle has different pressure-flow characteristics, showing no diastolic perfusion dominance like that seen in the left ventricle. ${ }^{19}$ The contraction forces and cavity pressures of the left ventricle and the right ventricle vary greatly, resulting in different intramyocardial compression effects on coronary vessel walls. Left coronary phasic flow shows strong flow impediment in systole; left coronary perfusion occurs mainly in diastole as a result of the distal suction effect produced by myocardial relaxation. ${ }^{16}$ In contrast, the systolic-diastolic pressure range of the right ventricle (approximately $0-25 \mathrm{~mm} \mathrm{Hg}$ ) is much lower than the aortic pressure range $(80-130 \mathrm{~mm} \mathrm{Hg})$. Blood thus is continuously driven by a high aortic pressure gradient into the right coronary artery during both right ventricular contraction and relaxation phases. WIA has recently been used to study right coronary arterial flow. ${ }^{20}$ The suction E5 wave was found to be less prominent than that of the left ventricle, and no diastolic dominance was seen in the proximal right coronary artery. ${ }^{20}$ IABP counterpulsation on right ventricular coronary perfusion thus would be expected to exhibit differences in augmentation characteristics. To our knowledge, there exists no counterpulsation investigation seeking to support optimization tailored to both left and right heart considerations. Further investigation will be important for understanding the variable impact of IABP counterpulsation in the presence of right ventricular failure; this type of investigation could be directly applicable to the care of patients with biventricular failure.

Any successful application of WIA for the diagnosis of pathologic arterial flows relies mainly on how precisely the wave speed is determined and how the forward and backward waves are split and interpreted. Current methods have used the single-point technique to determine wave speed in coronary arteries. ${ }^{12,16} \mathrm{We}$ used this method as the first of 2 methods to calculate wave speed, which has been shown to be appropriate in the setting of healthy coronary arteries (as was the case in this study). ${ }^{12,16}$ There are limitations, however, with this traditional single-point technique. Determination of coronary wave speed by single-point measurement has encountered paradoxic predictions during diseased conditions. ${ }^{15}$ For example, according to the sum-of-squares method, the predicted wave speed downstream of a proximal stenosis revascularized by a stent was found to be decreased rather than increased. ${ }^{15}$ Erroneous single-point wave speed estimates have also been found for hyperemic coronary flow with distal microvascular vasodilation induced by adenosine administration. ${ }^{15}$

Because our study was of nondiseased coronary arteries, the traditional method of calculating coronary wave speed with the single-point technique was still appropriate. In this study, however, we did introduce another way of calculating coronary wave speed by means of an in-housederived model-based identification method. ${ }^{11}$ This method was derived from the first principles of flow conservation laws and produces nonparadoxic results in diseased arteries, yielding results in close agreement with simulation data in validation trials and with traditional methods of coronary and aortic wave speed calculations in nondiseased arteries. ${ }^{3,12}$ Future research should continue to examine how 
best to determine coronary wave speed, especially in diseased arteries and in interventions commonly used in the catheter laboratory setting.

\section{CONCLUSIONS}

Wave intensities in the ascending aorta and left anterior descending coronary artery provide a mechanism to explain diastolic augmentation and systolic unloading of the IABP on the left side of the heart. In the aorta, during diastolic augmentation, IABP inflation generates a backward compression wave, which exerts a pushing effect toward the heart that translates to a forward compression wave into the coronary circulation. During systolic unloading, IABP deflation generates a backward expansion wave, which sucks blood from the left ventricle into the aorta. Although this backward expansion wave translates to a reduced left ventricular afterload, the sucking effect results in coronary blood steal, as seen by a forward expansion wave in the left anterior descending coronary flow. The waves are sensitive to inflation-deflation timing, with timing at the $\mathrm{R}$ wave, just $25 \mathrm{~ms}$ delayed from standard timing leading to a much weaker forward expansion wave and less coronary regurgitation.

We are grateful for Dr Willard Daggett's insight and mentorship on this project.

\section{References}

1. Papaioannou TG, Stefanadis C. Basic principles of the intraaortic balloon pump and mechanisms affecting its performance. ASAIO J. 2005;51:296-300.

2. Bolooki H. Clinical application of the intra-aortic balloon pump. 3rd ed. Armonk (NY): Futura; 1998

3. Kolyva C, Pantalos GM, Giridharan GA, Pepper JR, Khir AW. Discerning aortic waves during intra-aortic balloon pumping and their relation to benefits of counterpulsation in humans. J Appl Physiol. 2009;107:1497-503.

4. Sarnoff SJ, Braunwald E, Welch GH Jr, Case RB, Stainsby WN, Marcruz R. Hemodynamic determinants of oxygen consumption of the heart with special reference to the tension-time index. Am J Physiol. 1958;192:148-56.

5. Barnea O, Moore TW, Dubin SE, Jaron D. Cardiac energy considerations during intraaortic balloon pumping. IEEE Trans Biom Eng. 1990;37:170-81.
6. Zelano JA, Li JKJ, Welkowitz W. A closed-loop control scheme for intraaortic balloon pumping. IEEE Trans Biom Eng. 1990;37:182-92.

7. Parher KH, Jones CJ. Forward and backward running waves in the arteries: analysis using the method of characteristics. J Biomech Eng. 1990;112:322-6.

8. Parker KH. An introduction to wave intensity analysis. Med Biol Eng Comput. 2009; 47:175-88

9. Bleasdale RA, Parker KH, Jones CJ. Chasing the wave. Unfashionable but important new concepts in arterial wave travel. Am J Physiol Heart Circ Physiol. 2003;284:H1879-85

10. Lu PJ, Lin PY, Yang CF, Hung CH, Chan MY, Hsu TC. Hemodynamic and metabolic effects of para- versus intraaortic counterpulsatile circulation supports. ASAIO J. 2011;57:19-25.

11. Hung $\mathrm{CH}$. Analysis of phasic coronary arterial flow and wave characteristics using hybrid circulation model [thesis]. Tainan, Taiwan: National Cheng Kung University; 2008.

12. Davies JE, Whinnett ZI, Francis DP, Willson K, Foale RA, Malik IS, et al. Use of simultaneous pressure and velocity measurements to estimate arterial wave speed at a single site in humans. Am J Physiol Heart Circ Physiol. 2006;290:H878-85.

13. Siebes M, Kolyva C, Verhoeff BJ, Piek JJ, Spaan JA. Potential and limitations of wave intensity analysis in coronary arteries. Med Biol Eng Comput. 2009;47: 233-9.

14. Khir AW, O'Brien A, Gibbs JS, Parker KH. Determination of wave speed and wave separation in the arteries. J Biomech. 2001;34:1145-55.

15. Kolyva C, Spaan JAE, Piek JJ, Siebes M. Windkesselness of coronary arteries hampers assessment of human coronary wave speed by single-point technique. Am J Physiol Heart Circ Physiol. 2008;295:H482-90.

16. Davies JE, Whinnett ZI, Francis CH, Manisty CH, Aguado-Sierra J, Willson K et al. Evidence of a dominant backward-propagating "suction" wave responsible for diastolic filling in humans, attenuated in left ventricular hypertrophy Circulation. 2006;113:1768-78

17. Sun YH, Anderson TJ, Parker KH, Tyberg JV. Wave-intensity analysis: a new approach to coronary hemodynamics. J Appl Physiol. 2000;89:1636-44.

18. Koenig SC, Litwak KN, Giridharan GA, Pantalos GM, Dowling RD, Prabhu SD, et al. Acute hemodynamic efficacy of a 32-ml subcutaneous counterpulsation device in a calf model of diminished cardiac function. ASAIO J. 2008;54:578-84.

19. Heller LI, Silver KH, Villegas BJ, Balcom SJ, Weiner BH. Blood flow velocity in the right coronary artery: assessment before and after angioplasty. J Am Coll Cardial. 1994;24:1012-7.

20. Hadjiloizou N, Davies JE, Malik IS, Aguado-Sierra J, Willson K, Foale RA, et al Differences in cardiac microcirculatory wave patterns between the proximal left mainstem and proximal right coronary artery. Am J Physiol Heart Circ Physiol. 2008;295:H1198-205.

21. Sugawara M, Niki K, Ohte N, Okada T, Harada A. Clinical usefulness of wave intensity analysis. Med Biol Eng Comput. 2009;47:197-206.

22. Zambanini A, Cunningham SL, Parker KH, Khir AW. McG Thom SA, Hughes AD. Wave-energy patterns in carotid, brachial, and radial arteries: a noninvasive approach using wave-intensity analysis. Am J Physiol Heart Circ Physiol. 2005; 289:H270-6. 